\title{
The translationally controlled tumor protein TCTP is involved in cell cycle progression and heat stress response in the bloodstream form of Trypanosoma brucei
}

\author{
Borka Jojic ${ }^{1}$, Simona Amodeo ${ }^{1,2}$ and Torsten Ochsenreiter ${ }^{1, *}$ \\ ${ }^{1}$ Institute of Cell Biology, University of Bern, Bern, Switzerland. \\ 2 Graduate School for Cellular and Biomedical Sciences, University of Bern, Bern, Switzerland. \\ * Corresponding Author: \\ Torsten Ochsenreiter, Institute of Cell Biology, University of Bern, Bern, Switzerland; E-mail: torsten.ochsenreiter@izb.unibe.ch
}

\begin{abstract}
The translationally controlled tumor protein TCTP, is a universally conserved protein that seems to be of essential function in all systems tested so far. TCTP is involved in a multitude of cellular functions including cell cycle control, cell division, apoptosis and many more. The mechanism of how TCTP is involved in most of these functions remains elusive. Here we describe that TCTP is a cytoplasmic protein involved in cell cycle regulation and heat stress response in the bloodstream form of Trypanosoma brucei.
\end{abstract}

\author{
doi: $10.15698 / \mathrm{mic} 2018.10 .652$ \\ Received originally: 22.05.2018; \\ In revised form: 24.07.2018, \\ Accepted 26.07.2018, \\ Published 24.08.2018.
}

Keywords: TCTP, Trypanosoma brucei, mitochondria, heat stress response, acidocalcisomes.

\author{
Abbreviations: \\ BSF - bloodstream form, \\ PCF - procyclic form, \\ TCTP - translationally controlled \\ tumor protein.
}

\section{INTRODUCTION}

Trypanosoma brucei is a single celled protozoan parasite and the causative agent of human African sleeping sickness and Nagana in cattle. The parasite belongs to the Excavata, an eukaryotic supergroup only distantly related to the mainstream model systems within the Opisthokonta and as such represents a versatile model to study universally conserved proteins and their potential functions in biology.

The translationally controlled tumor protein (TCTP) is highly conserved among eukaryotes and is involved in a large variety of processes like cell growth and development, the cell cycle, apoptosis and the protection against cellular stresses, including oxidative stress and heat stress [1-8]. Moreover, several interacting/binding partners such as the elongation factor eEF-1alpha [9], tubulin [10], calcium [11] or $\mathrm{Na}^{+} / \mathrm{K}^{+}$-ATPase [12] have been identified.

We recently showed that $T$. brucei contains two isoforms TCTP1 and TCTP2 that are exclusively expressed in the procyclic form (PCF) and bloodstream form (BSF) parasites, respectively [13]. TCTP1 and TCTP2 have identical 5'UTRs and ten nucleotide changes in the open reading frames (ORFs) that lead to five amino acid changes. The exclusive expression is based on the different nucleotide composition of the 3'UTRs of the two isoforms, responsible for the different mRNA stabilities [13]. TCTP1 is localized in the cytoplasm of PCF cells and loss of the protein causes a growth defect and leads to several phenotypes including a decrease in number and enlargement of acidocalcisomes (ACs) as well as changes in mitochondrial morphology. Furthermore, the cells depleted of TCTP display asymmetrical cell divisions leading to the accumulation of shorter "tadpole" like cells [13]. While the cytoplasmic localization of TCTP has been described in several systems, the protein is also associated with other structures in the cell. In HeLa cells for example TCTP was mostly found in the nucleus, where it seems to be involved in anti-apoptotic activities since depletion by RNAi led to massive cell death by apoptosis [14]. Also, associations with mitochondria have previously been demonstrated, albeit mainly under stress conditions [15-17]. TCTP has previously also been described to be secreted and involved in inflammatory response through the release of histamine. How TCTP is released out of the cells remains enigmatic, but interestingly a similar observation was recently reported in trypanosome infected tsetse flies were TCTP might be involved in manipulating the microbiota of the fly [18].

As a consequence of harboring only one mitochondrion per cell with a singular mitochondrial genome known as the kinetoplast, trypanosomes display a synchronized mitochondrial and nuclear genome replication and segregation 
[19]. Mitochondrial genome replication initiates prior to nuclear DNA replication and also the segregation of the replicated mitochondrial genome occurs prior to mitosis. A wild type BSF population contains about $80-85 \%$ cells with one kinetoplast and one nucleus (1K1N), $10-15 \%$ cells with two kinetoplasts and one nucleus (2K1N) and up to $5 \%$ cells with two kinetoplasts and two nuclei $(2 \mathrm{~K} 2 \mathrm{~N})$ stage. Here we provide the first evidence for the localization of TCTP in BSF cells and its requirement for proper cytokinesis and heat stress response in the mammalian infective form of the parasite.

\section{RESULTS \\ TCTP2 localization}

In biochemical fractionations using digitonin and differential centrifugation followed by western blotting with the previously described anti-TCTP antibody, the protein is localized to the cytoplasmic fraction (Figure 1A). Since the antibody did not provide specificity in immunofluorescence microscopy, we tagged TCTP2 N- (myc) and C-terminally (triple HA) to evaluate its localization using anti-myc and anti-HA antibodies (Figure 1B). These experiments support the

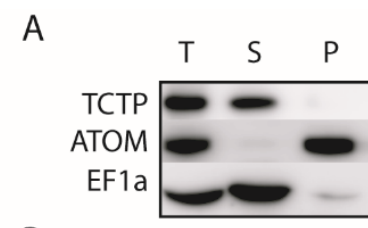

B
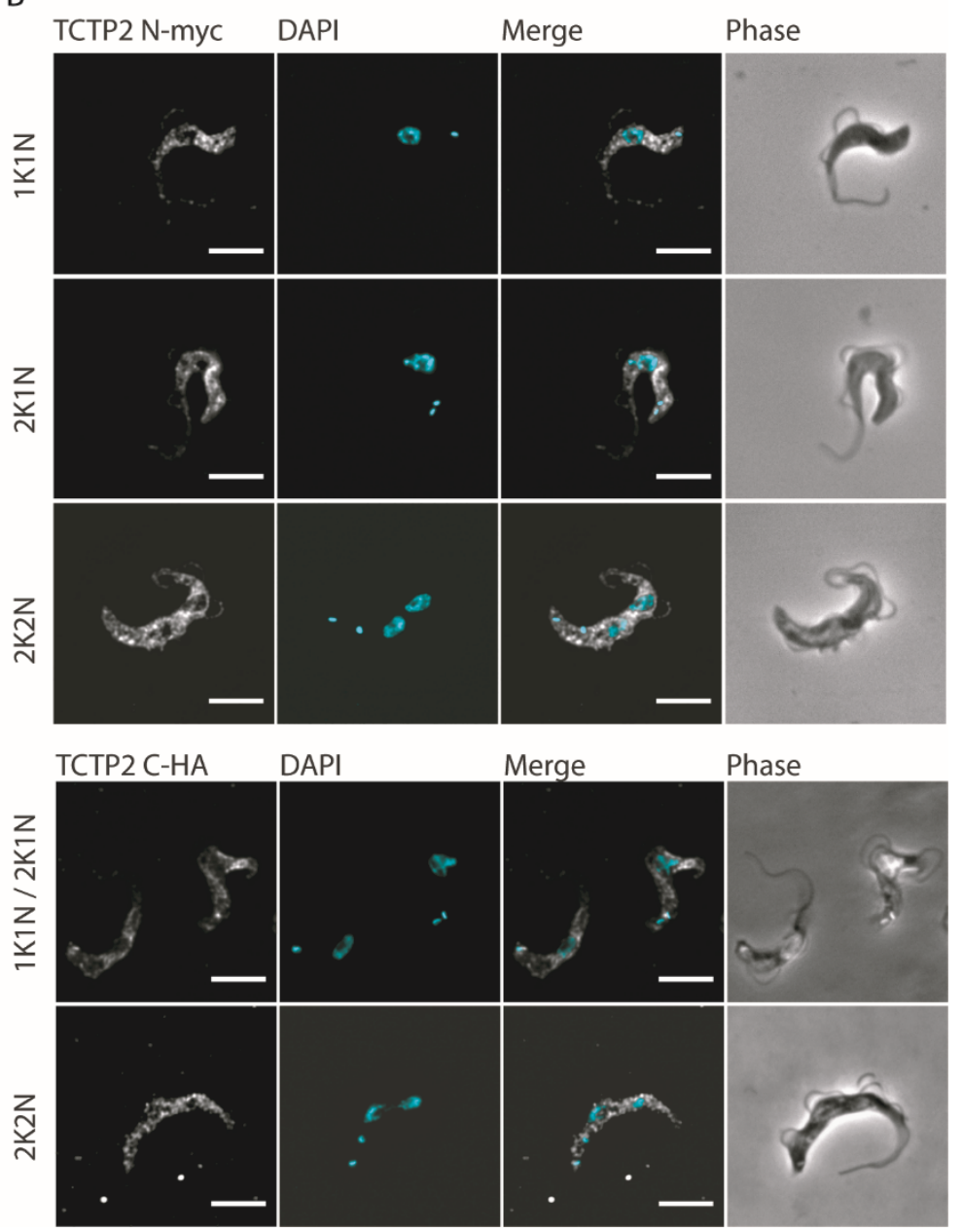

FIGURE 1: Localization of TCTP in bloodstream trypanosomes. (A) Western blot of BSF cells extracted with $0.025 \%$ digitonin. Total cellular extract (T), supernatant (S) and pellet (P) fractions were analysed by western blot decorated with antibodies against TCTP, ATOM and EF1 $\alpha$. (B) Immunofluorescence images of BSF cell expressing N-terminally myc-tagged TCTP2 or C-terminal HA-tagged TCTP2. Myc-TCTP2 was detected with anti-myc antibody (white). The DNA is detected by DAPI (cyan). K, kinetoplast DNA; N = nuclear DNA; Phase, phase contrast microscopy; scale bars: $5 \mu \mathrm{m}$. 
A

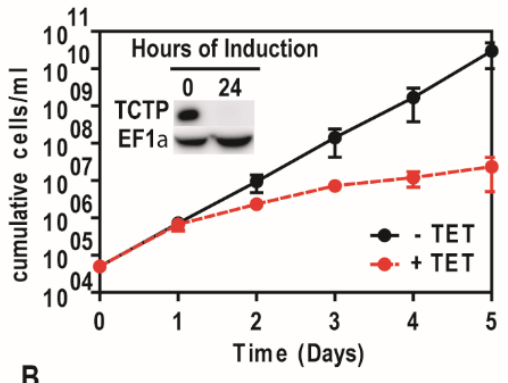

B

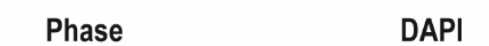

.
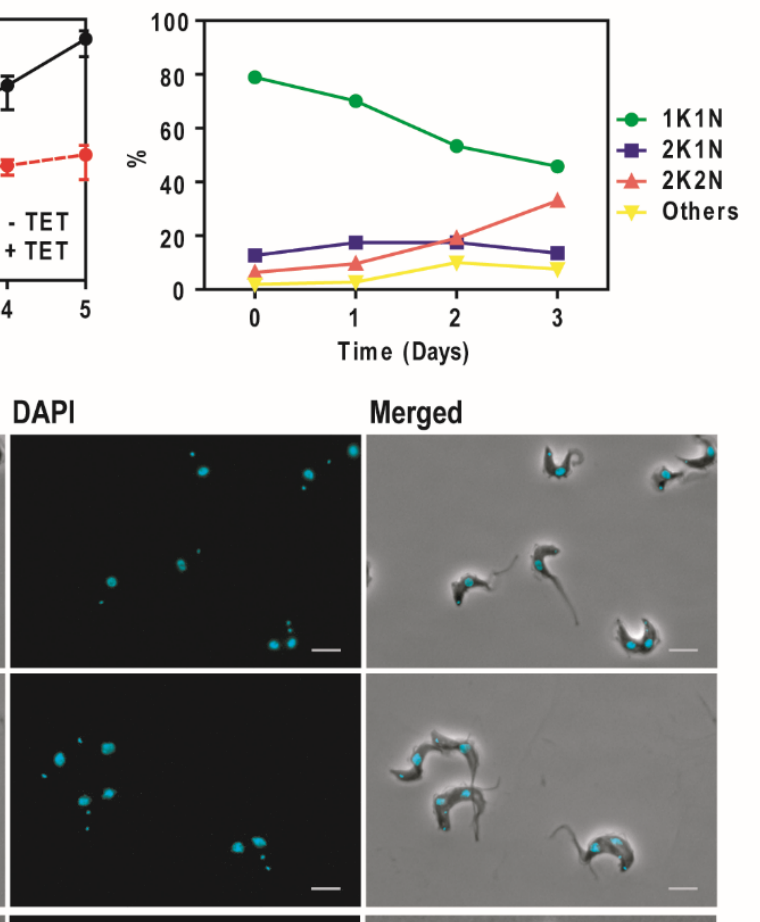

Merged
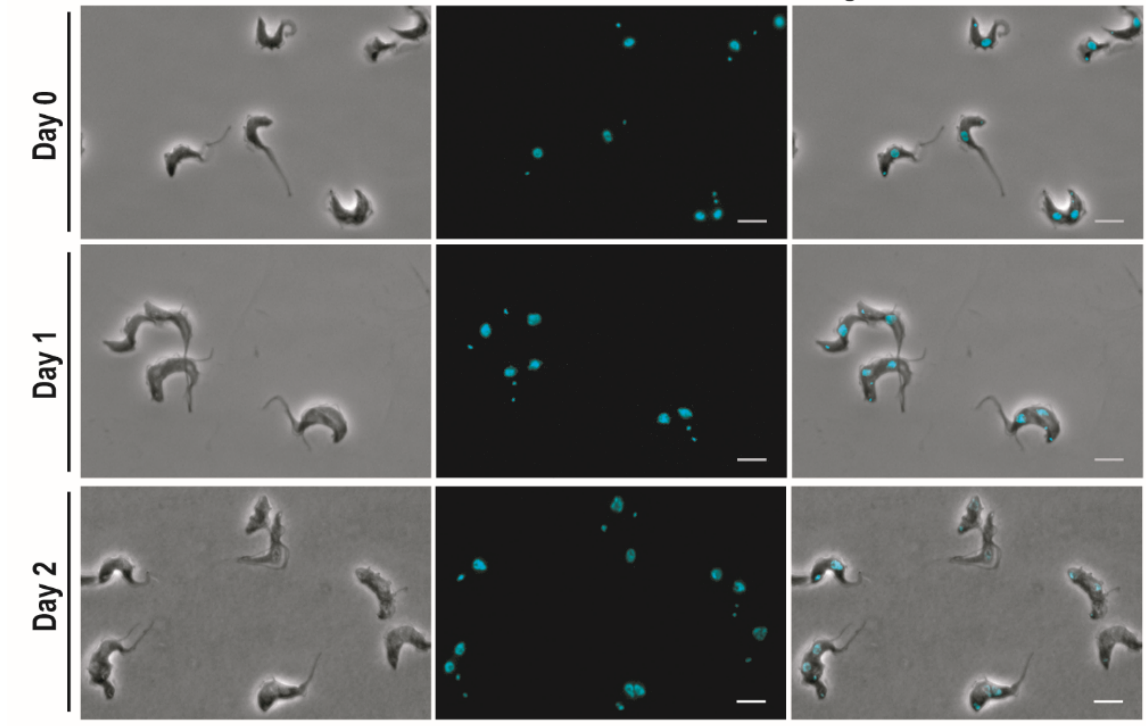

D
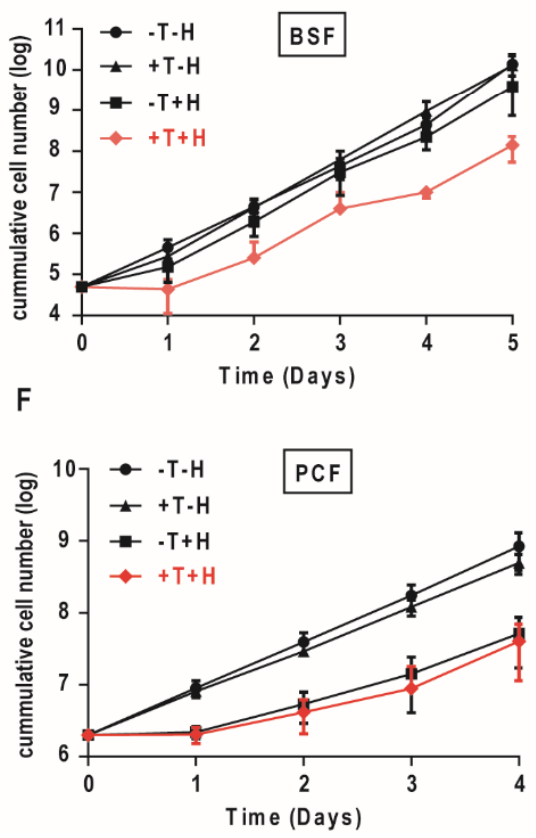

E

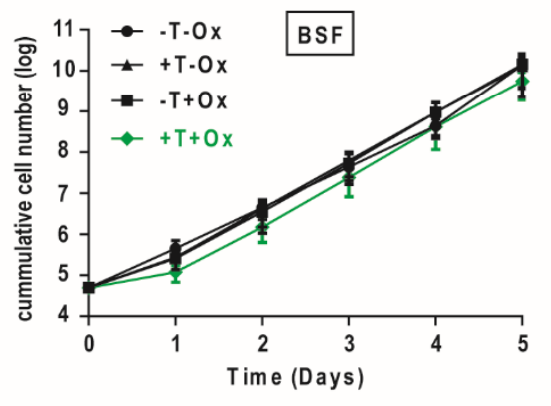

FIGURE 2: Effect of TCTP depletion, heat and oxidative stress in bloodstream trypanosomes. (A) Growth of cells with or without tet was monitored for five consecutive days. Bars represent standard deviation of four independent experiments. Inset: western blot showing TCTP downregulation upon 24 hours of RNAi induction. (B) Phase contrast and DAPI images of non-induced (Day 0 ) and induced (Day 1 and Day 2) bloodstream trypanosomes showing the effect of TCTP RNAi in cell morphology and cell cycle progression. (C) K-N counts in percentage following TCTP RNAi ( $n \geq 100$ ). Scale bars: $5 \mu \mathrm{m}$. (D, E, F) Monitoring growth of $T$. brucei upon TCTP RNAi followed by heat/oxidative stress. (D) BSF trypanosomes were heat shocked (+tet+heat shock, $+\mathrm{T}+\mathrm{H}$, red) at $42^{\circ} \mathrm{C}$ for one hour or (E) incubated with $50 \mu \mathrm{M}$ sodium hydrogen arsenate (+T+oxidative stress, $+\mathrm{T}+\mathrm{Ox}$, green) for three hours, or (F) PCF cells were subjected to 45 minutes heat shock at $41^{\circ} \mathrm{C}$. Subsequently the cells were washed in PBS and cultured again in $\mathrm{HMI}$ $9+10 \%$ FCS. Cell recovery was monitored for five consecutive days. Values represent averages from three independent experiments and error bars indicate standard deviation. Non-induced cells (-tet, $-T), 24$ hours induced (+tet, $+\mathrm{T}$ ) cells and noninduced cells but stressed respectively with sodium hydrogen arsenate $(-T+O x)$ or heat shock $(-\mathrm{T}+\mathrm{H})$ were used as controls. 
biochemical analysis and show that the ectopically expressed $\mathrm{N}$ - or C-terminally tagged TCTP2 is predominantly localized in the cytoplasm with a distinct depletion of the TCTP2 signal in the region of the nucleus (Figure 1B). Furthermore, the protein does not seem to change its localization during the cell cycle (Figure 1B). DAPI was used to stain nuclear and kinetoplast DNA.

\section{TCTP is required for normal cell growth and cell cycle pro- gression}

An inducible TCTP RNAi construct targeting the TCTP open reading frames was stably integrated in a BSF $T$. brucei cell line. We targeted the TCTP ORF in order to compare the results to the previously published phenotype in the PCF, where we also targeted the TCTP ORF. Depletion of TCTP mRNAs was induced through the addition of tetracycline (tet) and confirmed by western blot decorated with TCTP and EF1 $\alpha$ antibodies (Figure 2A; Inset). After 24 hours of RNAi induction, no TCTP could be detected by western blot. Cell growth was monitored for five consecutive days (Figure $2 A)$. Two days post RNAi induction the cells started to grow slower and the growth retardation became more severe on the following days. After five days of TCTP depletion the doubling time was prolonged from six to 24 hours, when compared to the non-induced cells. Phase contrast and DAPI images were used for cell morphology and cell cycle stage analysis (Figure 2B). Over the course of TCTP depletion, we observed an accumulation of cells in the $2 \mathrm{~K} 2 \mathrm{~N}$ stage of the cell cycle. Quantification of kinetoplast-nucleus counts showed that after three days of RNAi induction the number of cells with 2K2N DNA content increases from $4 \%$ to $33 \%$ ( $n \geq 100$; Figure $2 \mathrm{C}$ ). This corresponds to a decrease of cells in $\mathrm{G} 1$ of the cell cycle $(1 \mathrm{~K} 1 \mathrm{~N}$ ) from $80 \%$ to $50 \%$ (Figure $2 \mathrm{C}$ ). We did not observe a significant change in the number of $2 \mathrm{~K} 1 \mathrm{~N}$ cells. Phase contrast images did not reveal any obvious change in the cell morphology upon TCTP depletion.

\section{TCTP is involved in heat-stress response in BSF parasites}

Based on the reports in other model organisms we tested if TCTP in BSF cells is involved in stress response [20]. For this we induced TCTP RNAi for 24 hours in bloodstream trypanosomes. At this time the levels of TCTP in BSF are below detection limit and no growth phenotype is observed (see Figure 2A). Following the depletion of TCTP the cells were exposed to heat stress for one hour, then the cells were washed with PBS and cultured in HMI-9 with $10 \%$ FCS without tetracycline. The recovery of cell growth was monitored in both cases by following cell growth for five consecutive days (Figure 2D). As controls we used cells that were heat stressed but not induced with tetracyclin as well as cells where RNAi was induced but that were not stressed. Additionally, we also used oxidative stress induced by $\mathrm{NaAsO}_{2}$ (Figure 2E) and heat stress in the PCF (Figure 2F). Average cumulative values from three independent experiments were plotted using GraphPrism with error bars indicating the standard deviation. We observed that in the control experiments the BSF cells grew at a wild type rate of approximately four cell divisions per day, while the BSF cells exposed to heat shock after TCTP RNAi showed a delayed recovery by two days. In the first 24 hours post heat shock the cells depleted of TCTP did not grow and a normal growth rate was first observed between 48 and 72 hours post heat shock (Figure 2D). The BSF cells exposed to oxidative stress following depletion of TCTP (Figure 2E) had only one cell division in the first 24 hours of the recovery but regained the normal growth rate (four divisions in 24 hours) two days after the stress was induced. In the PCF cells the heat stress delayed growth for 24 hours, however no significant difference between TCTP depleted cells and the wild type situation was observed. Based on these experiments we suggest that TCTP depletion in BSF cells has only a minor impact on recovery following oxidative stress while it is more pronounced under heat stress. In PCF cells recovery from heat stress does not seem to be influenced by the lack of TCTP.

TCTP depletion does not alter mitochondrial or acidocalcisome morphology

To verify if the depletion of TCTP in BSF trypanosomes would lead to mitochondrial structure abnormalities such as the ones observed in the PCF cells [13] we induced TCTP RNAi in BSF cells and visualized the mitochondria using the mitochondrial heat shock protein 70 (mtHSP-70) as a marker. Immunofluorescence images show no aberration in the mitochondrial structure upon two days of TCTP depletion (Figure 3A). Similarly, we asked if TCTP depletion in BSF cells would change the number and/or morphology of acidocalcisomes as we had previously observed in PCF cells. For this we visualized the acidocalcisomes by immunofluorescence microscopy using the vacuolar proton pyrophosphatase (VP1) antibody (Figure 3B, [21]. The DNA and cell morphology were visualized by DAPI and phase contrast images. Following three days of TCTP depletion, we did not notice enlarged acidocalcisomes or a significant change in their number (Figure 3B, C).

\section{Identification of possible interactors and binding proteins of TCTP}

In order to identify possible targets or interactors of TCTP in BSF trypanosomes we analysed the whole cell proteome before and after TCTP depletion using stable isotope labelling with amino acids in cell culture (SILAC) combined with mass spectrometry (Figure S1A-C, [22-24]). Samples form non-induced and induced bloodstream trypanosomes were mixed in 1:1 ratio (cell number) and analyzed by liquid chromatography mass spectrometry. Overall, we could detect the changes of 2557 proteins. Proteome changes upon TCTP depletion for 24, 36 and 48 hours are presented by volcano plots (Figure S1A-C). Each plot represents the proteome comparison between non-induced cells and the respective time point of TCTP RNAi induction. In each plot the $x$-axes represent the proteins abundance change ( $\log _{2}$ fold change) while the $y$-axes represent the significance of the observed fold change ( $\log _{10} p$-value). Marked in red is TCTP2. Over the course of TCTP RNAi induction, we observed a progressive depletion of TCTP2. We did not detect other proteins whose abundance changed progressively and significantly in all timepoints during the course of TCTP RNAi. 
A
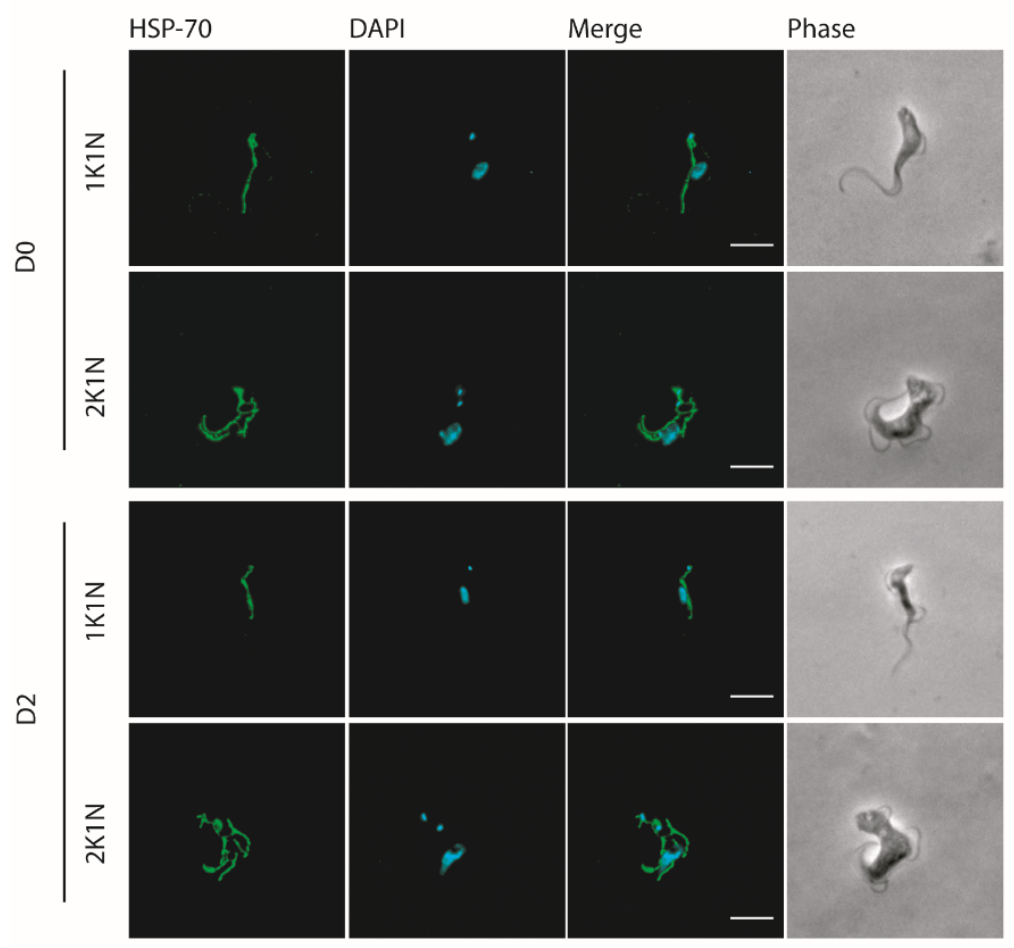

B VP1

DAPI

Merge
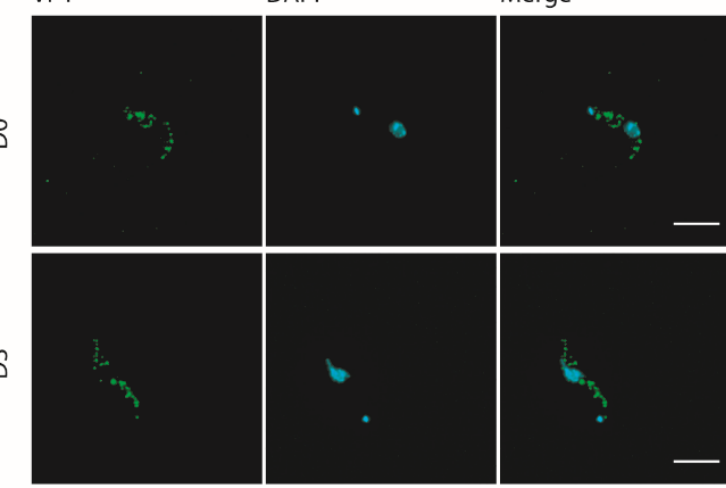

Phase

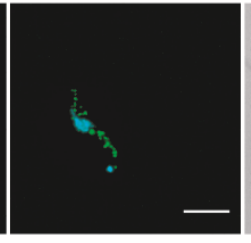

C

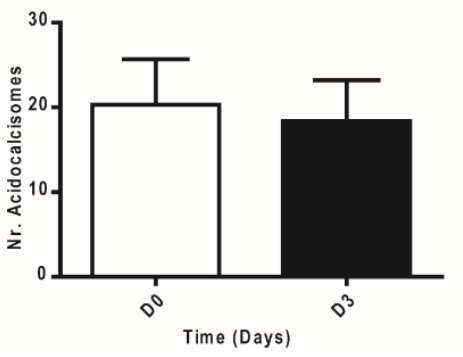

Similarly, we did not identify any interaction partners of the PCF TCTP1 (Figure S2) when performing pull-down with the c-terminally HA-tagged version.

\section{DISCUSSION}

We have previously reported that the $T$. brucei genome encodes two paralogues of the translationally controlled

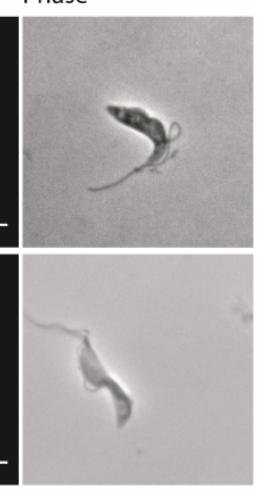

FIGURE 3: Effect of TCTP depletion on mitochondria and acidocalcisomes in BSF cells. (A) Immunofluorescence images of noninduced (D0) and induced at day two (D2) bloodstream cells stained for mitochondria (HSP-70, green). (B) Morphology of acidocalcisomes following downregulation of TCTP in BSF. Non-induced (DO) and induced at day three (D3) BSF trypanosomes were stained for acidocalcisome with the marker VP1 (green). Nuclear DNA and kDNA were detected with DAPI (cyan). Phase contrast images (grey) show cell morphology. Cell morphology is shown by phase contrast images ( $\mathrm{PH}$, grey). (C) Histograms showing the mean number of acidocalcisomes per cell in D0 and D3 cells ( $n$ =13). Scale bars: $5 \mu \mathrm{m}$. 
controlling differential expression and characterized the morphological and organelle phenotypes in the insect form [13]. Here, we study the function of TCTP in the BSF cells and compare them to the PCF providing a more comprehensive picture of TCTP in T. brucei (Table 1).

The localization of TCTP2 in the BSF is very similar to the insect form (Table 1). Based on biochemical and imaging data the protein is mostly distributed throughout the cytoplasm with a distinct depletion in the nucleus. Also, the localization does not change during the BSF cell cycle, similar to what we reported for the PCF trypanosomes [13]. A cytoplasmic localization in trypanosomes is consistent with most reports from plant, yeast or mammalian systems [16, 25]. However, some reports have shown nuclear localization in human cells [26], as well as transient association with mitochondria and microtubules in yeast cells $[16,25]$.

In order to test function of TCTP in BSF cells we used RNAi targeting the TCTP ORF. In previous experiments we specifically depleted the BSF TCTP2 by targeting the TCTP2 3'UTR, which led to a growth retardation, and while the overall TCTP levels remained below detection limit in western blotting, the exclusive depletion of TCTP2 was accompanied by a temporary increase of the levels of TCTP1 mRNA [13] (Table 1). Interestingly, RNAi experiments targeting the ORF of TCTP, thus also affecting the residual TCTP1 expression, resulted in a much stronger growth inhibition of BSF cells. This supports the previously suggested hypothesis that the residual expression of TCTP1 in BSF cells might partially compensate for the specific loss of TCTP2.

In BSF cells we detect a specific increase in the $2 \mathrm{~K} 2 \mathrm{~N}$ cells during TCTP ORF RNA, while specific depletion of TCTP2 leads to a slight increase in $1 \mathrm{~K} 1 \mathrm{~N}$ cells (Figure S3) as was seen for the TCTP depletion in PCF cells. Thus, the specific depletion of the BSF TCTP2 shows a different phenotype than depletion of both paralogues suggesting different functions of the two proteins. Alternatively, the ORF RNAi might be more efficient in depleting TCTP2 since it covers a larger region of the transcript, and this might lead to the different phenotypes.
The $2 \mathrm{~K} 2 \mathrm{~N}$ cells have undergone mitochondrial and nuclear DNA replication as well as segregation but seem to be blocked or delayed in cytokinesis. Interestingly, this phenotype was not observed in the BSF specific TCTP2 RNAi knockdown (see Figure $\mathrm{S} 3$ and [13]). Here a small increase in $1 \mathrm{~K} 1 \mathrm{~N}$ cells similar to the PCF TCTP knockdown described previously was observed [13]. While in BSF parasites cytokinesis is blocked or delayed, the cell division in PCF cells is unequal with one daughter inheriting a not fully developed posterior end leading to a "tadpole" like morphology (Table 1). A precytokinesis arrest similar to the one described above was previously observed by Sheader and coworkers upon silencing of VSG in BSF cells. Here the number of $2 \mathrm{~K} 2 \mathrm{~N}$ cells reached up to $60 \%$ at 48 hours post induction [27]. The authors speculated that this checkpoint which seems absent in the PCF cells is required in vivo to respond to potential changes in VSG levels during cell division [27].

For example, TCTP transiently interacts with microtubules and numerous cell cycle proteins including the polo-like kinese-1 [28], the checkpoint protein Chfr [29], the mitotic regulator $\mathrm{Cdc} 25 \mathrm{C}$ [8] and nucleolar proteins [30]. These proteins interfere in different steps of the cell cycle resulting in a broad number of phenotypes when alternating the levels of TCTP. In this study we aimed to identify TCTP binding partners or effectors using SILAC proteomics after TCTP depletion. We only included timepoints before the growth phenotype appeared in order to avoid the detection of secondary effects. While we could clearly detect the loss of TCTP as a consequence of the TCTP RNAi we did not detect any other significant changes. Thus, TCTP might be involved in maintaining functionality or localization of its interacting partners rather than simply increasing the stability.

TCTP has been described as a calcium binding protein for the first time 20 years ago in trypanosomes [11] and later confirmed also in other organisms [31]. We recently demonstrated that TCTP depletion in PCF parasites causes alterations in the morphology of the major calcium storage organelles, acidocalcisomes and mitochondria [13]. In BSF cells both organelles seem not affected by the loss of TCTP,

TABLE 1. Comparison of TCTP1 and TCTP2 in PCF and BSF parasites, respectively.

\begin{tabular}{|c|c|c|}
\hline & PCF & BSF \\
\hline Expressed paralogue & TCTP1 & TCTP2 \\
\hline Localization & Cytoplasmic & Cytoplasmic \\
\hline \multicolumn{3}{|c|}{ TCTP1/2 depletion by RNAi } \\
\hline Cell growth & Growth inhibition & Strong growth inhibition \\
\hline Cell cycle & No change & Increase in $2 \mathrm{~K} 2 \mathrm{~N}$ cells \\
\hline Cell morphology & Tadpole morphology & No change \\
\hline Acidocalcisomes & Enlarged in size and reduced in number & No change \\
\hline Mitochondria & Accumulations in the network & No change \\
\hline + heat stress & No change & Decreased growth \\
\hline + oxidative stress & NA & No change \\
\hline Cell growth & Growth inhibition & Strong growth inhibition \\
\hline
\end{tabular}


while the growth defect remained. Thus, potentially the effect on the calcium storage organelles in PCF are due to secondary effects.

One of the best studied and conserved functions of TCTP is the ability to protect cells against a vast number of cellular stresses. During heat shock, an elevated level of TCTP has been observed in worm parasites such as Schistosoma and Trichinella $[6,7]$. This has been hypothesised to act as a stress defence mechanism to protect the parasite during the transition from the cold-blooded vectors (snails) to the worm blooded hosts (vertebrates). On the other hand, silencing of the gene using RNAi led to decreased tolerance to cold and high temperatures in the Brassica oleracea cabbage [5]. Here we tested whether the presence of TCTP helps in cell recovery following heat or oxidative stresses. For this we depleted TCTP in BSF cells and subjected them to the heat or oxidative stress, followed by monitoring the cell growth recovery. We found that the cells in which TCTP was depleted prior to the heat stress survive the stress but recover with a delay of two days compared to the controls. Interestingly this result was observed only in bloodstream cells and not in PCF indicating that stress response might be specific for that life cycle stage. Following the mild oxidative stress, both non-induced and induced cells recovered at similar rates. Overall, these data suggest that endogenous levels of TCTP are required for the bloodstream forms recovery following heat stress. TCTP might be chaperoning other proteins and/or mRNAs which help cellular recovery, a model already suggested in the worm parasites [6].

\section{MATERIALS AND METHODS}

\section{Trypanosome cell lines and culturing}

For RNAi and gene tagging experiments we used transgenic $T$. brucei bloodstream (New York single marker, NYsm) cell lines co-expressing T7 RNA polymerase and a tet repressor [32]. The BSF cells were cultured at $37^{\circ} \mathrm{C}$ and $5 \% \mathrm{CO}_{2}$ in HMI-9 medium supplemented with $10 \%$ FCS [33] in the presence of $2.5 \mu \mathrm{g} / \mathrm{ml}$ geneticin (G418).

\section{Plasmid constructs and transfection}

For inducible RNAi against TCTP mRNAs we used a pLEW100 based stem-loop plasmid [32,34] where an insert of 512 bp targeting the full ORF sequence of the TCTP2 gene was integrated. The constructs were linearized with Notl and $10 \mu \mathrm{g}$ were transfected in NYsm BSF by electroporation. The positive clones were selected with blasticidin $(2.5 \mathrm{~g} / \mathrm{ml}$ in BSF). Induction of RNAi was done by addition of $1 \mu \mathrm{g} / \mathrm{ml}$ tet. For the C-terminal tagging one allele of TCTP2 in BSF was in situ tagged with a triple Hemagglutinin (HA) epitope [35]. For inducible $\mathrm{N}$-terminal c-Myc tagging, the full ORF plus the first 21 nt from the 3'UTR of TCTP2 were amplified by PCR and cloned in pJM-2 vector (a gift from A. Schneider; [34]. Upon transfection (as described above) the clones were selected with puromycine. Expression was induced by addition of $1 \mathrm{\mu g} / \mathrm{ml}$ tet.

\section{Growth recovery assay}

Downregulation of TCTP in BSF and PCF was induced for 24 hours. The cells were then washed twice in PBS and subjected to either heat or oxidative stress. For the heat shock, the cells were pelleted by centrifugation $(2500 \mathrm{rpm} / 8 \mathrm{~min})$, re- suspended and incubated in pre-warmed media (one hour at $42^{\circ} \mathrm{C}$ HMI-9 for BSF, 45 minutes at $41^{\circ} \mathrm{C}$ SDM-79 for PCF). Oxidative stress was induced by incubating BSF for 3 hours in HMI-9 media supplemented with $50 \mu \mathrm{M}$ sodium arsenate, $\mathrm{NaAsO}_{2}$ [36]. Immediately after each stress the cells were washed in PBS and re-cultured in their normal growth conditions. Their recovery was monitored by counting cell growth in the next four or five consecutive days. Average cumulative values from three independent experiments were plotted using GraphPrism with error bars indicating the standard deviation.

\section{Western blot}

For western blotting trypanosome pellets were washed in phosphate buffered saline (PBS, $\mathrm{pH}=7.2$ ), re-suspended in standard Laemmli buffer (LB) $\left(10^{6}\right.$ in $\left.15 \mu \mathrm{l}\right)$, boiled for $5 \mathrm{~min}$ at $95^{\circ} \mathrm{C}$ and cooled on ice for 5 minutes. For the digitonin fractionation the cells were washed once in PBS, then the pellets were resuspended in SoTE buffer (0.6 M sorbitol, $2 \mathrm{mM}$ EDTA, $20 \mathrm{mM}$ Tris- $\mathrm{HCl}, \mathrm{pH} 7.5$ ) containing $0.025 \%$ digitonin and incubated on ice for $5 \mathrm{~min}$. The cell fractions were then separated by differential centrifugation at $8000 \mathrm{rcf}$ for $5 \mathrm{~min}$ at $4^{\circ} \mathrm{C}$. The fractions were lysed in $\mathrm{LB}$, boiled for $5 \mathrm{~min}$ at $95^{\circ} \mathrm{C}$, cooled on ice for 5 minutes and loaded on $10 \%$ or $12 \%$ SDS-polyacrylamide gels (10 $-10^{7}$ cells per lane) before subjected to western blotting. The proteins were transferred onto PVDF Immobilon-P membranes (Millipore) using BioRad wet blotting system, blocked for 1 hour at room temperature in 10\% skimmed milk or BSA solution in PBST (PBS + 0.1\% TWEEN-20) and decorated with the primary antibodies. In this study we used rat-polyclonal anti-TCTP (1:50, Eurogentech), rabbit anti-ATOM (1:10000, [37]) and mouse anti-EF1alpha (1:10000, SantaCruz). After washing the primary antibody by incubating the membranes 3 times, 10 minutes each time in PBST, the membranes were incubated for 1 hour at room temperature with the secondary antibody. Secondary antibodies were: rabbit anti-rat HRP-conjugate (1:10000, Dako), swine anti-rabbit HRP-conjugate (1:10000, Dako) and rabbit anti-mouse HRP-conjugate (1:10000, Dako). For the chemiluminescent detection, the SuperSignal system (Pierce) was used and images were acquired with Amersham Imager 600.

\section{Immunofluorescence and microscopy}

For immunofluorescence, BSF were harvested by slow centrifugation (2000 rpm/5 min), washed once in PBS and then fixed on slides for 4 min with $4 \%$ PFA in PBS. The cells were permeabilized for 5 min with $0.2 \%$ TritonX-100 in PBS and blocked for 30 min with 4\% BSA in PBS. The cells were incubated for 1 hour in a wet chamber with primary antibody diluted in $4 \%$ BSA in PBS, washed three times in PBST and incubated again in dark wet chambers with secondary antibodies diluted in $4 \%$ BSA in PBS. The cells were mounted with ProLong ${ }^{\circledR}$ Gold Antifade Mountant with or without DAPI (Invitrogen). Images were acquired with Leica DM 5500 fluorescent light microscope and/or Leica SP8 Confocal Microscope System with STED and deconvoluted by Leica LAS AF and Huygens software, respectively. The primary antibodies used in this study were: rabbit anti-myc (1:1000, Sigma), rabbit anti-HA (1:1000, Sigma), mouse antimtHSP70 (1:2000, [38]) and rabbit anti-VP1 (1:2000, [21]). Secondary antibodies were goat anti-rabbit IgG, goat anti-mouse IgG conjugated with fluorophores Alexa Fluor ${ }^{\circledR} 488$, Alexa Fluor $^{\circledR} 594$ (1:1000, Invitrogen). 


\section{ACKNOWLEDGEMENTS}

We acknowledge the proteomics \& mass spectrometry core facility at the Department of BioMedical Research, University of Bern for help with proteomics. Hélène Baudouin and Anneliese Hoffmann for discussion.

\section{CONFLICT OF INTEREST}

The authors declare no conflict of interest.

\section{COPYRIGHT}

(C) 2018 Jojic et al. This is an open-access article released under the terms of the Creative Commons Attribution (CC BY)

\section{REFERENCES}

1. Hsu Y-C, Chern JJ, Cai Y, Liu M, and Choi K-W (2007). Drosophila TCTP is essential for growth and proliferation through regulation of $d R h e b$ GTPase. Nature 445(7129): 785-8. doi: 10.1038/nature05528

2. Chen SH, Wu P-S, Chou C-H, Yan Y-T, Liu H, Weng S-Y, and Yang-Yen $\mathrm{H}-\mathrm{F}$ (2007). A knockout mouse approach reveals that TCTP functions as an essential factor for cell proliferation and survival in a tissue- or cell type-specific manner. Mol Biol Cell 18(7): 2525-32. doi: 10.1091/mbc.E07-02-0188

3. Berkowitz O, Jost R, Pollmann S, and Masle J (2008). Characterization of TCTP, the translationally controlled tumor protein, from Arabidopsis thaliana. Plant Cell 20(December): 3430-3447. doi: 10.1105/tpc.108.061010

4. Gnanasekar M, and Ramaswamy K (2007). Translationally controlled tumor protein of Brugia malayi functions as an antioxidant protein. Parasitol Res 101(6): 1533-1540. doi: 10.1007/s00436-007-0671-z

5. Cao B, Lu Y, Chen G, and Lei J (2010). Functional characterization of the translationally controlled tumor protein (TCTP) gene associated with growth and defense response in cabbage. Plant Cell Tissue Organ Cult 103(2): 217-226. doi: 10.1007/s11240-010-9769-6

6. Gnanasekar M, Dakshinamoorthy G, and Ramaswamy K (2009). Translationally controlled tumor protein is a novel heat shock protein with chaperone-like activity. Biochem Biophys Res Commun 386(2): 333-7. doi: 10.1016/j.bbrc.2009.06.028

7. Mak CH, Su KW, and Ko RC (2001). Identification of some heatinduced genes of Trichinella spiralis. Parasitology 123(Pt 3): 293-300. PMID: 11578093

8. Chan THM, Chen L, Liu M, Hu L, Zheng BJ, Poon VKM, Huang P, Yuan YF, Huang JD, Yang J, Tsao GSW, and Guan XY (2012). Translationally controlled tumor protein induces mitotic defects and chromosome missegregation in hepatocellular carcinoma development. Hepatology 55(2): 491-505. doi: 10.1002/hep.24709

9. Langdon JM, Vonakis BM, and MacDonald SM (2004). Identification of the interaction between the human recombinant histamine releasing factor/translationally controlled tumor protein and elongation factor-1 delta (also known as eElongation factor-1B beta). Biochim Biophys Acta - Mol Basis Dis 1688(3): 232-236. doi: 10.1016/j.bbadis.2003.12.007

10. Tuynder M, Susini L, Prieur S, Besse S, Fiucci G, Amson R, and Telerman A (2002). Biological models and genes of tumor reversion: cellular reprogramming through tpt1/TCTP and SIAH-1. Proc Natl Acad Sci U S A 99(23): 14976-14981. doi: 10.1073/pnas.222470799

11. Haghighat NG, and Ruben $L$ (1992). Purification of novel calcium binding proteins from Trypanosoma brucei: properties of 22-, 24- and 38-kilodalton proteins. Mol Biochem Parasitol 51(1): 99-110. doi: 10.1016/0166-6851(92)90205-X license, which allows the unrestricted use, distribution, and reproduction in any medium, provided the original author and source are acknowledged.

Please cite this article as: Borka Jojic, Simona Amodeo and Torsten Ochsenreiter (2018). The translationally controlled tumor protein TCTP is involved in cell cycle progression and heat stress response in the bloodstream form of Trypanosoma brucei. Microbial Cell 5(10): 460-468. doi: 10.15698/mic2018.10.652

12. Jung J, Kim M, Kim M-J, Kim J, Moon J, Lim J-S, Kim M, and Lee K (2004). Translationally controlled tumor protein interacts with the third cytoplasmic domain of $\mathrm{Na}, \mathrm{K}-\mathrm{ATP}$ ase alpha subunit and inhibits the pump activity in HeLa cells. J Biol Chem 279(48): 49868-75. doi: 10.1074/jbc.M400895200

13. Jojic B, Amodeo S, Bregy I, and Ochsenreiter T (2018). Distinct $3^{\prime}$ UTRs regulate the life-cycle-specific expression of two TCTP paralogs in Trypanosoma brucei. J Cell Sci 131(9). doi: 10.1242/jcs.206417

14. Li F, Zhang D, and Fujise K (2001). Characterization of fortilin, a novel antiapoptotic protein. J Biol Chem 276(50): 47542-9. doi: 10.1074/jbc.M108954200

15. Rid R, Önder K, Trost A, Bauer J, Hintner H, Ritter M, Jakab M, Costa I, Reischl W, Richter K, Macdonald S, Jendrach M, Bereiter-Hahn J, and Breitenbach M (2010). H 2 O 2 -dependent translocation of TCTP into the nucleus enables its interaction with VDR in human keratinocytes: TCTP as a further module in calcitriol signalling. J Steroid Biochem Mol Biol J Steroid Biochem Mol Biol 118: 29-40. doi: 10.1016/j.jsbmb.2009.09.015

16. Rinnerthaler $M$, Jarolim S, Heeren G, Palle $E$, Perju $S$, Klinger $H$, Bogengruber E, Madeo F, Braun RJ, Breitenbach-Koller L, Breitenbach M, and Laun P (2006). MMI1 (YKL056c, TMA19), the yeast orthologue of the translationally controlled tumor protein (TCTP) has apoptotic functions and interacts with both microtubules and mitochondria. Biochim Biophys Acta 1757(5-6): 631-8. doi: 10.1016/j.bbabio.2006.05.022

17. Diraison F, Hayward K, Sanders KL, Brozzi F, Lajus S, Hancock J, Francis JE, Ainscow E, Bommer $\mathrm{U}$ a, Molnar E, Avent ND, and Varadi a (2011). Translationally controlled tumour protein (TCTP) is a novel glucose-regulated protein that is important for survival of pancreatic beta cells. Diabetologia 54(2): 368-79. doi: 10.1007/s00125-010-19587

18. Bossard G, Bartoli M, Fardeau M-L, Holzmuller P, Ollivier B, and Geiger A (2017). Characterization of recombinant Trypanosoma brucei gambiense Translationally Controlled Tumor Protein (rTbgTCTP) and its interaction with Glossina midgut bacteria. Gut Microbes 8(5): 413-427. doi: 10.1080/19490976.2017.1331833

19. Gluenz E, Povelones ML, Englund PT, and Gull K (2011). The kinetoplast duplication cycle in Trypanosoma brucei is orchestrated by cytoskeleton-mediated cell morphogenesis. Mol Cell Biol 31(5): 101221. doi: 10.1128/MCB.01176-10

20. Rinnerthaler M, Lejskova R, Grousl T, Stradalova V, Heeren G, Richter K, Breitenbach-Koller L, Malinsky J, Hasek J, and Breitenbach M (2013). Mmi1, the Yeast Homologue of Mammalian TCTP, Associates with Stress Granules in Heat-Shocked Cells and Modulates Proteasome Activity. PLoS One 8(10): e77791. doi: 10.1371/journal.pone.0077791 
21. Rodrigues CO, Scott DA, and Docampo R (1999). Characterization of a vacuolar pyrophosphatase in Trypanosoma brucei and its localization to acidocalcisomes. Mol Cell Biol 19(11): 7712-23. PMID: 10523660

22. Gunasekera K, Wüthrich D, Braga-Lagache S, Heller M, and Ochsenreiter $T$ (2012). Proteome remodelling during development from blood to insect-form Trypanosoma brucei quantified by SILAC and mass spectrometry. BMC Genomics 13: 556. doi: 10.1186/1471-216413-556

23. Cirovic O, and Ochsenreiter T (2014). Whole proteome analysis of the protozoan parasite trypanosoma brucei using stable isotope labeling by amino acids in cell culture and mass spectrometry. Methods Mol Biol 1188: 47-55. doi: 10.1007/978-1-4939-1142-4_4

24. Dejung M, Subota I, Bucerius F, Dindar G, Freiwald A, Engstler M, Boshart M, Butter F, and Janzen CJ (2016). Quantitative Proteomics Uncovers Novel Factors Involved in Developmental Differentiation of Trypanosoma brucei. PLoS Pathog 12(2): e1005439. doi: 10.1371/journal.ppat.1005439

25. Acunzo J, Baylot V, So A, and Rocchi P (2014). TCTP as therapeutic target in cancers. Cancer Treat Rev 40(6): 760-769. doi: 10.1016/j.ctrv.2014.02.007

26. Zhang J, de Toledo SM, Pandey BN, Guo G, Pain D, Li H, and Azzam EI (2012). Role of the translationally controlled tumor protein in DNA damage sensing and repair. Proc Natl Acad Sci U S A 109(16): E926-33. doi: 10.1073/pnas.1106300109

27. Sheader K, Vaughan S, Minchin J, Hughes K, Gull K, and Rudenko G (2005). Variant surface glycoprotein RNA interference triggers a precytokinesis cell cycle arrest in African trypanosomes. Proc Natl Acad Sci U S A 102(24): 8716-21. doi: 10.1073/pnas.0501886102

28. Yarm FR (2002). Plk Phosphorylation Regulates the MicrotubuleStabilizing Protein TCTP. Mol Cell Biol 22(17): 6209-21. doi: 10.1128/MCB.22.17.6209

29. Burgess A, Labbe J-C, Vigneron S, Bonneaud N, Strub J, Dorsselaer A Van, Lorca T, and Castro A (2008). Chfr interacts and colocalizes with TCTP to the mitotic spindle. Oncogene 27167: 5554-5566. doi: 10.1038/onc. 2008.167

30. Johansson H, Svensson F, Runnberg R, Simonsson T, and Simonsson $S$ (2010). Phosphorylated Nucleolin Interacts with Translationally Controlled Tumor Protein during Mitosis and with Oct4 during Interphase in ES Cells. PLoS One 5(10):e13678. doi: 10.1371/journal.pone.0013678
31. Bommer U-A, and Thiele B-J (2004). The translationally controlled tumour protein (TCTP). Int J Biochem Cell Biol 36: 379-385. doi: 10.1016/S1357-2725(03)00213-9

32. Wirtz E, Leal S, Ochatt C, and Cross GA (1999). A tightly regulated inducible expression system for conditional gene knock-outs and dominant-negative genetics in Trypanosoma brucei. Mol Biochem Parasitol 99(1): 89-101. PMID: 10215027

33. Hirumi H, and Hirumi K (1989). Continuous cultivation of Trypanosoma brucei blood stream forms in a medium containing a low concentration of serum protein without feeder cell layers. J Parasitol 75(6): 985-9. PMID: 2614608

34. Mani J, Desy S, Niemann M, Chanfon A, Oeljeklaus S, Pusnik M, Schmidt O, Gerbeth C, Meisinger C, Warscheid B, and Schneider A (2015). Mitochondrial protein import receptors in Kinetoplastids reveal convergent evolution over large phylogenetic distances. Nat Commun 6: 6646. doi: 10.1038/ncomms7646

35. Oberholzer M, Morand S, Kunz S, and Seebeck T (2006). A vector series for rapid PCR-mediated C-terminal in situ tagging of Trypanosoma brucei genes. Mol Biochem Parasitol 145(1): 117-20. doi: 10.1016/j.molbiopara.2005.09.002

36. Fernandez-Moya SM, Garcia-Perez A, Kramer S, Carrington M, and Estévez AM (2012). Alterations in DRBD3 Ribonucleoprotein Complexes in Response to Stress in Trypanosoma brucei. PLoS One 7(11): 1-10. doi: 10.1371/journal.pone.0048870

37. Pusnik M, Schmidt O, Perry AJ, Oeljeklaus S, Niemann M, Warscheid $B$, Lithgow T, Meisinger C, and Schneider A (2011). Mitochondrial preprotein translocase of trypanosomatids has a bacterial origin. Curr Biol 21(20): 1738-43. doi: 10.1016/j.cub.2011.08.060

38. Klein KG, Olson CL, and Engman DM (1995). Mitochondrial heat shock protein 70 is distributed throughout the mitochondrion in a dyskinetoplastic mutant of Trypanosoma brucei. Mol Biochem Parasitol 70(1-2): 207-9. PMID: 7637705 
